\title{
Comparative proteomic analysis of ductal breast carcinoma demonstrates an altered expression of chaperonins and cytoskeletal proteins
}

\author{
PAOLO CARCOFORO ${ }^{1}$, BLENDI URA ${ }^{5}$, CARLO MISCHIATI $^{2}$, MONICA SQUERZANTI ${ }^{2}$, VINCENZO LANZARA ${ }^{2}$, \\ CARLO CERVELLATI ${ }^{2}$, ROBERTA CALZA ${ }^{2}$, PATRIZIA POLVERINO DE LAURETO ${ }^{3}$, ERICA FRARE ${ }^{3}$, \\ MATTIA PORTINARI ${ }^{1}$, GIORDANA FERIOTTO ${ }^{1}$, SERENA LANZARA $^{1}$, \\ ENZO AGOSTINELLI ${ }^{4}$ and CARLO M. BERGAMINI ${ }^{2}$
}

\begin{abstract}
Departments of ${ }^{1}$ Morphology, Surgery and Experimental Medicine and ${ }^{2}$ Biomedical Sciences and Specialty Surgery,
University of Ferrara, Ferrara; ${ }^{3}$ CRIBI Biotechnology Centre, University of Padova, Padova;

${ }^{4}$ Istituto Pasteur-Fondazione Cenci Bolognetti, Department of Biochemical Sciences,

Sapienza University of Rome and CNR Institute of Molecular Biology and Pathology, Rome;

${ }^{5}$ Institute for Maternal and Child Health, IRCSS Burlo Garofolo, Trieste, Italy
\end{abstract}

Received January 7, 2013; Accepted March 7, 2013

DOI: $10.3892 / \mathrm{mmr} .2013 .1375$

\begin{abstract}
The aim of the present study was to analyze the protein composition of ductal breast carcinoma and the surrounding normal tissue in individual patients using comparative $2 \mathrm{D}$ proteomics and mass spectrometry to detect candidate disease biomarkers for diagnosis and prognosis. Samples of normal and cancerous tissue obtained form 28 patients were analyzed. Chaperonins and cytoskeletal proteins predominated among the 11 proteins for which major changes in abundance were detected. Of these 11 proteins with an altered expression, 2 had a decreased expression and 9 had an increased expression. In addition, the abundance of a few cytokeratins was also altered; however, they were not capable of serving as specific circulatory biomarkers. The proteins which we observed to exhibit an altered expression in infiltrating ductal breast carcinoma may be exploited as novel targets for therapeutic interventions or represent novel diagnostic/prognostic markers for the early detection of aggressive tumors, particularly those with multridrug-resistant phenotypes during the earlier stages of the disease.
\end{abstract}

\section{Introduction}

With an incidence of approximately 1 million new cases each year, breast cancer (BC) constitutes a major cause of mortality

Correspondence to: Professor Carlo Mischiati, Department of Biomedical Sciences and Specialty Surgery, Section of Biochemistry, Clinical Biochemistry and Molecular Biology, University of Ferrara, Via Luigi Borsari 46, I-44100 Ferrara, Italy

E-mail:msc@unife.it

Key words: proteomics, breast cancer, cancer biomarkers in females diagnosed at the stage of infiltrating disease (1). BC is classically classified as either lobular or ductal in form, with scirrhous, medullary and mucinous variants. Their biological and clinical heterogeneity and variable response to therapy lead to refined classifications based on receptor status (2) as luminal A and B type [estrogen receptor (ER)-positive]; human epidermal growth factor receptor (HER)2 overexpressing epidermal growth factor (EGF) receptors; and basal type [not expressing ER, progesterone (PR) and HER, also known as triple-negative breast cancer (TNBC)] and normallike cancer. Attempts to devise additional classifications are based on markers for diagnosis and prognosis (cytokeratins and chaperonins), in relation to specific mutations detected by proteomic and cDNA microarray techniques (3). Such studies are currently in progress.

In the present study, we performed a proteomic analysis of normal and $\mathrm{BC}$ tissue from individual patients undergoing mastectomy at the Ferrara University Hospital (Ferrara, Italy), during the last 2 years. Changes in the expression of specific proteins in the majority of patients support the investigation of their role as tissue and serological markers for the identification of aggressive tumors and as targets for therapy refractory cases.

\section{Materials and methods}

Tissue specimens. Samples of normal and cancerous tissue were collected from 28 patients (represented as P1 to P28) with ductal BC for proteomic analysis. The study was approved by the Institutional Ethics Committee of the Ferrara University Hospital. Diagnosis was confirmed by histopathological analysis, which demonstrated that the tumor specimens contained $>50 \%$ tumor cells. Samples from 10 patients providing large quantities of tissue were snap-frozen in liquid nitrogen and stored at $-80^{\circ} \mathrm{C}$ until proteomic analysis was performed. 


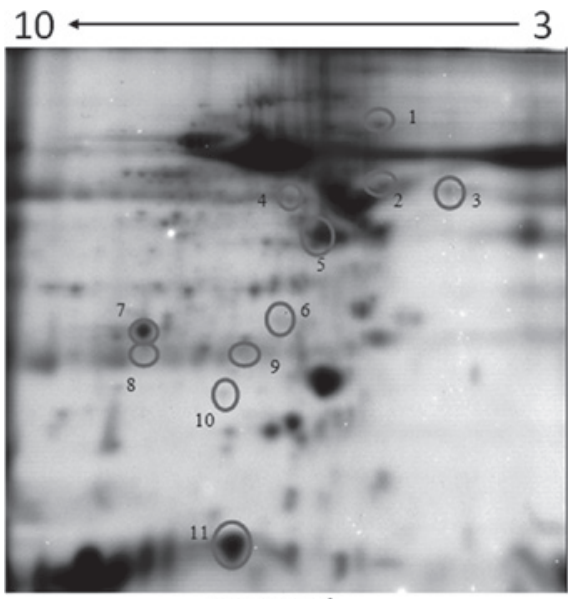

normal

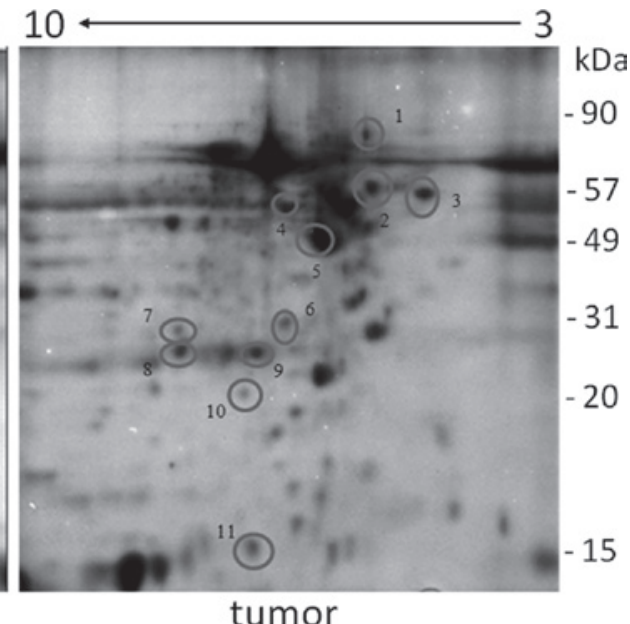

tumor

Figure 1. Two dimensional (2D) gel electrophoresis of mammary tissue proteins. Total protein from homogenates of tumor or normal breast tissues from the same patient were loaded onto each gel and separated by $2 \mathrm{D}$ gel electrophoresis. The spots circled in the image represent the relevant upregulated or downregulated proteins, which were excised and analyzed by liquid chromatography-mass spectrometry (LC-MS)/MS. The identified proteins are listed in Table I.

Proteomic analysis by 2 dimensional (2D) electrophoresis and mass spectrometry. Proteomic analysis of BC and normal tissue from individual patients was performed by homogenization in 2.5 volumes of $7 \mathrm{M}$ urea, $2 \mathrm{M}$ thiourea, 4\% CHAPS, $40 \mathrm{mM}$ Tris, $1 \mathrm{mM}$ benzamidine, $1 \mathrm{mM}$ iodacetamide and $1 \mathrm{mM}$ EDTA, pH 8.8. Following centrifugation, portions of supernatant corresponding to $260 \mu \mathrm{g}$ protein were separated by isoelectric focusing (IEF) on precast pH 3-10 linear IPG strips at $40,000 \mathrm{Vh}$, according to the manufacturer's instructions. Following thiol reduction by $1 \%$ dithiothreitol (DTT) and alkylation by $4 \%$ iodoacetamide, the strips were separated on 2D SDS-PAGE on $12.5 \%$ polyacrylamide gels for $1 \mathrm{~h}$ at 200 V. Gels were stained with colloidal Coomassie, scanned with the Molecular Imager PharosFX System and analyzed using the ProteomeWeaver 4 program (Bio-Rad, Hercules, CA, USA). Spots excised from the gels were processed by trypsin digestion for mass spectrometry-based peptide identification. The gel fragments were briefly rinsed in buffered acetonitrile and then dried. Following thiol group reduction and alkylation, the peptides were digested overnight with $12.5 \mathrm{ng} / \mu$ l trypsin, resuspended in aqueous formic acid and analyzed with an Ultimate 3000 Nano/micro HPLC apparatus coupled with a LTQ-Orbitrap XL mass spectrometer (Thermo Scientific, Sunnyvale, CA, USA) (4,5). Using Excalibur 2.0.7 software, spectra were submitted for peptide identification to the MASCOT program against the NCBI database.

\section{Results}

The clinical characteristics of each individual patient associated with altered protein expression are summarized in Table I. The proteins with altered expression are also listed, as recognized by $2 \mathrm{D}$ gel electrophoresis. We achieved optimal resolution at a total load of $260 \mu \mathrm{g}$ of protein on the IEF strip, running 2D electrophoresis at a constant voltage of $200 \mathrm{~V}$ for $1 \mathrm{~h}$ in order to avoid proteins $<20 \mathrm{kDa}$ running out of the gel. Typically, 2D electrophoresis of tumor and normal samples of individual patients (Fig. 1) resolved several hundreds of proteins. By visual inspection, 11 proteins had altered expres- sion in BC tissue (circles, Fig. 1); 2 proteins had a decreased expression and 9 had an increased expression. These proteins were identified by mass spectrometry. The 9 proteins with an increased expression (Table I) were endoplasmin precursor (gp96), protein disulfide isomerase (PDI), TCP-1 subunit $\theta$ (TCP1- $\theta$ ), F actin-capping protein subunit $\beta$ (FACP- $\beta$ ), heat shock protein $\beta 1$ (namely, HSP27), triosephosphate isomerase (TPI), RS/DJ-1, $\beta$-tubulin (Tub- $\beta$ ) and $\beta$-actin (Act- $\beta$ ), while carbonic anhydrase-1 (CA-1) and adipocyte fatty acid binding-protein (A-FABP) were downregulated. These proteins included molecular chaperones, cytoskeletal proteins and metabolic enzymes. We also identified several cytokeratin peptides; however, these were ignored due to possible contamination during sample processing. Cytokeratins are relevant in BC immunocytochemical approaches, however, not when searching for circulating biomarker discovery (6).

The estimation of the abundance of proteins with altered expression was achieved through normalization of the intensity of spots from different gels against a virtual spot calculated as an average on the same gel of 5-6 spots with identical expression in all analyzed samples. Ratios of proteins present were obtained in spots of BC and corresponding normal tissue in 2D electrophoresis gels (Table I). Ratios $>2$ or $<0.5$ denote proteins significantly upregulated or downregulated in BC versus normal tissue, respectively. Thus, gp96 and TPI proteins were consistently modulated in at least 7 out of 10 patients, even if the score for TPI should be higher due to its expression not being constantly detectable in normal tissue, thus precluding the calculation of a precise ratio. PDI, FACP- $\beta$ and Tub- $\beta$ were highly expressed in 8 out of 10 tumors. HSP27, RS/DJ-1, CA-1 and A-FABP were consistently modulated in 9 out of 10 patients. Act- $\beta$ and TCP1- $\theta$ were elevated in all tumors analyzed.

\section{Discussion}

In the present study, the abundance of numerous proteins in normal and cancerous breast tissue was analyzed, with 11 proteins exhibiting a consistently altered expression in 


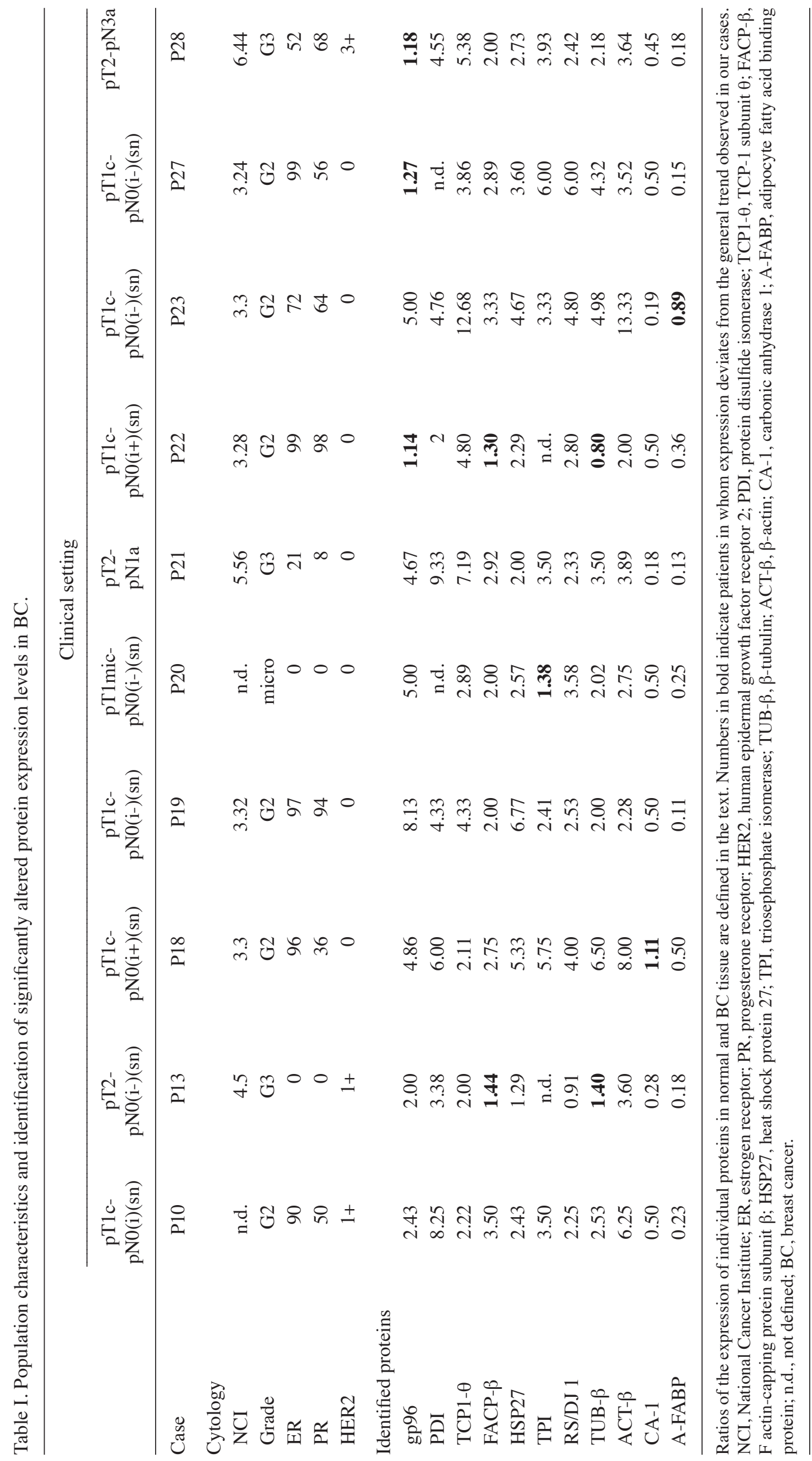


tumors. The proteins which exhibited an altered expression were as follows:

i) Chaperonins, which are notably involved in increased tumorigenicity, metastatic potential and resistance to chemotherapy. Chaperonins include HSP27 induced under unfavorable conditions to protect cells from death, preventing aggregation of denatured proteins, regulating caspase activity, intracellular redox state, polymerization of actin and cytoskeletal dynamics (7). HSP27 is a proposed immunocytochemical discriminator to refine $\mathrm{C} 3$ and $\mathrm{C} 4$ categories in suspect $\mathrm{BC}$ aspirates (8) and gp96 of the HSP90 family (9), which represents a tool for active immunization against tumors by associating with cell surface peptides for presentation to cytolytic T lymphocytes and cell destruction. Notably Vitespen, a peptide vaccine based on gp96, prolongs survival in patients with early-stage melanoma or renal cancer (10). The presence of gp96 in infiltrating ductal $\mathrm{BC}$ is attractive for vaccine treatment in TNBC patients resistant to classical therapy while maintaining the expression of gp96 (patient P20).

ii) The cytoskeletal proteins, Act- $\beta$, FACP- $\beta$, Tub- $\beta$ and TCP1- $\theta$, of which the latter protein assists in the ATP-dependent folding of actin and tubulin (11). Notably, some cytoskeletal proteins upregulated in BC are involved in ER activation: Act- $\beta$ binds to the ER- $\alpha$ complex, contributing to ER nuclear functions (12); chaperonin TCP1- $\theta$ (spot 3) is involved in the folding of Act- $\beta$ (13) and estrogen-regulated HSP27 controls the palmitoylation of ER, which is required for its interaction with membranes (14). This indicates that cytoskeletal rearrangement is a key step in the motility mechanisms leading to metastatic spreading; however, it may also be involved in hormone receptivity (15).

iii) Signaling proteins, including RS/DJ-1, TPI and A-FABP. $\mathrm{RS} / \mathrm{DJ}-1$ is an oncogene protein regulating RNA protein interaction, present in sera from BC patients but not from healthy patients, along with circulating antibodies against this protein (16). Similarly, autoantibodies against TPI are present in BC patients (17) and also in patients with oral cavity and lung squamous cell carcinoma $(18,19)$. PDI catalyzes the formation and rearrangement of protein disulfide bonds, acting as a reductase at the cell surface cleaving disulfide bonds with structural modifications of cell-associated proteins and as chaperonin (inhibiting aggregation of misfolded proteins) or antichaperonin (facilitating aggregation) inside cells, depending on the concentration. In addition, PDI binds estrogen and thyroid hormones (20). Notably, the knockdown of PDI in MCF7 $\mathrm{BC}$ cells induces caspase-dependent apoptosis (21). A-FABP is another protein whose expression is negatively affected. It plays a role in intracellular lipid transport and metabolism as well as signaling. The prognostic value for A-FABP has been reported in bladder cancer since its decreased expression correlates with poor prognosis (22). It is induced by PPAR ligands [the promoter region of the A-FABP gene contains functional peroxisome proliferator-responsive elements (23)] and its overexpression is likely beneficial in the treatment of bladder cancer. Contrasting results have been reported in $\mathrm{BC}$ since serum levels of A-FABP have been associated with tumor risk and aggressive behavior (24); however, comparable values of expression have been reported in ductal infiltrating carcinoma and normal tissue (25). Our data instead report the decreased levels of A-FABP in ductal BC compared with normal tissues in 9 out of 10 patients, including the TNBC patient in our population. The association between $\mathrm{BC}$ and A-FABP, and if the data are confirmed in larger populations, the possible selective induction by PPAR ligands may impact on the development of future strategies for the treatment of TNBC.

In conclusion, despite the limited number of patients investigated in the present study, the robustness of our results suggests that similar results may be observed in future studies with a larger sample size, including other tumor types and their multidrug-resistant (MDR) counterparts. This is an aspect of particular interest, since one of the drawbacks of conventional anticancer therapy is the development of drug resistance. The proteins which we observed to exhibit an altered expression in infiltrating ductal BC may be exploited as novel targets for therapeutic interventions or represent novel diagnostic/prognostic markers for the early detection of aggressive tumors, particularly those with MDR phenotypes during the earlier stages of the disease.

\section{Acknowledgements}

This study was supported by grants from Fondazione Cassa Risparmio di Ferrara to Professor Paolo Carcoforo.

\section{References}

1. Pisani P, Bray F and Parkin DM: Estimates of the world-wide prevalence of cancer for 25 sites in the adult population. Int $\mathrm{J}$ Cancer 97: 72-81, 2002.

2. Sørlie T, Perou CM, Tibshirani R, et al: Gene expression patterns of breast carcinomas distinguish tumor subclasses with clinical implications. Proc Natl Acad Sci USA 98: 10869-10874, 2001.

3. Gast MC, Schellens JH and Beijnen JH: Clinical proteomics in breast cancer: a review. Breast Cancer Res Treat 116: 17-29, 2009.

4. Bozzo C, Spolaore B, Toniolo L, et al: Nerve influence on myosin light chain phosphorylation in slow and fast skeletal muscles. FEBS J 272: 5771-5785, 2005.

5. Shevchenko A, Tomas H, Havlis J, Olsen JV and Mann M: In-gel digestion for mass spectrometric characterization of proteins and proteomes. Nat Protoc 1: 2856-2860, 2006.

6. Galvão ER, Martins LM, Ibiapina JO, Andrade HM and Monte SJ: Breast cancer proteomics: a review for clinicians. J Cancer Res Clin Oncol 137: 915-925, 2011.

7. Acunzo J, Katsogiannou M and Rocchi P: Small heat shock proteins HSP27 (HspB1), $\alpha$ B-crystallin (HspB5) and HSP22 (HspB8) as regulators of cell death. Int J Biochem Cell Biol 44: 1622-1631, 2012.

8. Keeling J and McKee GT: Heat shock protein (HSP)27: a further refinement in the diagnosis of suspicious fine needle aspirates of breast. Cytopathology 10: 40-49, 1999.

9. Yang Y and Li Z: Roles of heat shock protein gp96 in the ER quality control: redundant or unique function? Mol Cells 20: 173-182, 2005.

10. Randazzo M, Terness P, Opelz G and Kleist C: Active-specific immunotherapy of human cancers with the heat shock protein Gp96-revisited. Int J Cancer 130: 2219-2231, 2012.

11. Brackley KI and Grantham J: Activities of the chaperonin containing TCP-1 (CCT): implications for cell cycle progression and cytoskeletal organisation. Cell Stress Chaperones 14: 23-31, 2009.

12. Ambrosino C, Tarallo R, Bamundo A, et al: Identification of a hormone-regulated dynamic nuclear actin network associated with estrogen receptor alpha in human breast cancer cell nuclei. Mol Cell Proteomics 9: 1352-1367, 2010.

13. Vainberg IE, Lewis SA, Rommelaere H, et al: Prefoldin, a chaperone that delivers unfolded proteins to cytosolic chaperonin. Cell 93: 863-873, 1998.

14. Razandi M, Pedram A and Levin ER: Heat shock protein 27 is required for sex steroid receptor trafficking to and functioning at the plasma membrane. Mol Cell Biol 30: 3249-3261, 2010. 
15. Jiang P, Enomoto A and Takahashi M: Cell biology of the movement of breast cancer cells: intracellular signalling and the actin cytoskeleton. Cancer Lett 284: 122-130, 2009.

16. Le Naour F, Misek DE, Krause MC, Deneux L, Giordano TJ, Scholl S and Hanash SM: Proteomics-based identification of RS/ DJ-1 as a novel circulating tumor antigen in breast cancer. Clin Cancer Res 7: 3328-3335, 2001.

17. Tamesa MS, Kuramitsu Y, Fujimoto M, et al: Detection of autoantibodies against cyclophilin A and triosephosphate isomerase in sera from breast cancer patients by proteomic analysis. Electrophoresis 30: 2168-2181, 2009.

18. Shukla S, Pranay A, D'Cruz AK, Chaturvedi P, Kane SV and Zingde SM: Immunoproteomics reveals that cancer of the tongue and the gingivobuccal complex exhibit differential autoantibody response. Cancer Biomark 5: 127-135, 2009.

19. Zhang XZ, Xiao ZF, Li C, et al: Triosephosphate isomerase and peroxiredoxin 6, two novel serum markers for human lung squamous cell carcinoma. Cancer Sci 100: 2396-2401, 2009.

20. Fu XM, Wang P and Zhu BT: Characterization of the estradiolbinding site structure of human protein disulfide isomerase (PDI). PLoS One 6: e27185, 2011.

21. Hashida T, Kotake Y and Ohta S: Protein disulfide isomerase knockdown-induced cell death is cell-line-dependent and involves apoptosis in MCF7 cells. J Toxicol Sci 1: 1-7, 2011.
22. Boiteux G, Lascombe I, Roche E, Plissonnier ML, Clairotte A, Bittard $\mathrm{H}$ and Fauconnet $\mathrm{S}$ : A-FABP, a candidate progression marker of human transitional cell carcinoma of the bladder, is differentially regulated by PPAR in urothelial cancer cells. Int J Cancer 124: 1820-1828, 2009.

23. Schachtrup C, Emmler T, Bleck B, Sandqvist A and Spener F: Functional analysis of peroxisome-proliferator-responsive element motifs in genes of fatty acid-binding proteins. Biochem J 382: 239-245, 2004.

24. Hancke K, Grubeck D, Hauser N, Kreienberg R and Weiss JM: Adipocyte fatty acid-binding protein as a novel prognostic factor in obese breast cancer patients. Breast Cancer Res Treat 119: 367-377, 2010.

25. Li H, Lu Q, Dong LH, Xue H, Zhou HY and Yang HJ: Expression of fatty acid binding protein in human breast cancer tissues. Xi Bao Yu Fen Zi Mian Yi Xue Za Zhi 23: 312-316, 2007.

26. Elston CW and Ellis IO: Pathological prognostic factors in breast cancer. I. The value of histological grade in breast cancer: experience from a large study with long-term follow-up. Histopathology 19: 403-410, 1991. 